\title{
USO DE PRONOMBRES DE PRIMERA PERSONA SINGULAR OMITIDOS Y EXPRESOS EN PRODUCCIONES DE APRENDICES DE ESPAÑOL*
}

\author{
Violeta Cautín-Epifani**
}

\begin{abstract}
Resumen
El presente artículo tiene como objetivo examinar el uso de pronombres personales de primera persona singular expresos y omitidos en producciones de aprendices de español. Nos centraremos principalmente en identificar los patrones pragmáticos de uso de estos pronombres de acuerdo a los postulados por Lubbers Quesada y Blackwell (2009). Nuestro corpus de investigación está constituido por las transcripciones de 18 entrevistas orales semidirigidas a alumnos de español norteamericanos de nivel intermedio. Nuestros resultados indican el uso preferente de pronombre omitido en este contexto, lo cual se condice con lo encontrado por otras investigaciones (Al-Kasey \& Pérez Leroux, 1998; Isabelli, 2004; Liceras, 1989; Lubbers Quesada \& Blackwell, 2009). Nuestros análisis también indican un sobreuso del pronombre expreso en situaciones de promiencia del referente, lo que no sería adecuado de acuerdo a las reglas reglas ya señaladas. Basándonos en estos resultados, se argumenta a favor de la aplicación del análisis pragmático para el área de aprendizaje de español ya que permitiría detectar con mayor fineza las necesidades o faltas de adecuación del uso de la lengua por parte de los aprendices.
\end{abstract}

Palabras clave: uso de pronombres personales, aprendizaje de español, análisis pragmático.

\section{USE OF NULL AND OVER FIRST PERSON PRONOUN IN ORAL PRODUCTION OF LEANERS OF SPANISH}

\begin{abstract}
The objective of the following article is to examine the use of null and overt first-person pronouns by speakers of Spanish. We will focus mainly on identifying the pragmatic patterns of use based on the postulates of Lubbers Quesada and Blackwell (2009). The corpus we researched is made up of 18 oral interviews carried out with American students learning Spanish. Our results indicate the preferential use of null pronouns, which agrees with what has been found in previous studies in different contexts (Al-Kasey \& Pérez Leroux, 1998; Isabelli, 2004; Liceras, 1989; Lubbers Quesada \& Blackwell, 2009). Finally, this investigation verifies the usefulness of pragmatic analysis in Spanish as an additional language studies, since it allows for the more accurate detection of learner needs in terms of the appropriate use of the language.
\end{abstract}

Keywords: personal pronoun use, Spanish studies, pragmatic analysis

Recibido: 02-09- 2014

Aceptado: 24-12-2014

* Artículo vinculado con la tesis doctoral en evaluación Formas de tratamiento en el discurso escrito en medios virtuales: una investigación en hablantes chilenos realizada con el apoyo de la Beca de Doctorado de la Comisión Nacional de Ciencia y Tecnología de Chile (CONICYT) y en el marco del proyecto FONDECYT 1140912 titulado "El discurso político de las comunidades virtuales de opinión en el espacio público de los portales electrónicos de la prensa chilena"

** Chilena, Doctora (c) en Lingüística de la Pontificia Universidad Católica de Valparaíso. Valparaíso, Chile. violeta.cautin@gmail.com 


\section{Introducción}

El español ha sido caracterizado como una lengua pro-drop (Chomsky, 1981), es decir, favorece la omisión de pronombres. En este sentido, el español se opone al inglés, lengua en la cual los pronombres de sujeto deben ser siempre expresados. Ahora bien, pese a que las investigaciones respecto del uso de estos pronombres por parte de aprendices de español como lengua extranjera han arrojado que los estudiantes sí son capaces de incorporar la noción de la omisión de pronombres en su interlengua, también se ha encontrado que todavía persiste la dificultad por parte de estos aprendices de utilizar adecuadamente los pronombres omitidos y expresos a nivel pragmático y discursivo (Al-Kasey \& Pérez Leroux, 1998; Isabelli, 2004; Liceras, 1989; Lubbers Quesada \& Blackwell, 2009; Moreno, 2011; Pladevall, 2013; Saunders, 1999).

Según diversos autores, el uso de pronombre personal ya sea expreso $\mathrm{u}$ omitido en español por parte de hablantes nativos no es del todo opcional ni tampoco completamente equivalente. Los hablantes nativos perciben diferencias en su utilización en la mayoría de los contextos (Luján, 1999). Incluso se han detectado diferencias de uso de estos pronombres en relación a su expresión y omisión, así como a su posición pospuesta o antepuesta de acuerdo a factores de tipo cognitivo, pragmático, textual y dialectal (Aijón, Machado \& Serrano, 2010; Aijón \& Serrano, 2012; Orozco \& Guy, 2008; Serrano \& Aijón, 2010a; Serrano \& Aijón, 2010b; Serrano, 2012; Serrano, 2014)

En el habla oral se ha descrito el uso de pronombre personal expreso como la forma marcada, ya que la forma que se privilegia sería su omisión (Blackwell, 1998; 2003). Serrano (2014) postula que el uso del pronombre expreso 'yo' se relaciona con la manifestación de la subjetividad en el discurso, más frecuente en textos de tipo argumentativo.

De este mismo modo, en relación a la expresión del pronombre 'yo' acompañando al verbo creer, Serrano y Aijón (2010a) postulan que su omisión es más recurrente en contextos en los que se pretende proyectar objetividad profesional, dejando su expresión para contextos relacionados con la subjetividad y argumentación, tal como lo mencionamos anteriormente. Debido a su variabilidad, la enseñanza y adquisición de este rasgo se vuelve un problema tanto para los aprendices de español como lengua extranjera como para sus profesores, quienes no siempre 
cuentan con la competencia pragmática necesaria para enseñar su correcta utilización.

En atención a esta problemática, nos hemos propuesto en el presente trabajo analizar el uso de pronombres personales de primera persona singular por parte de estudiantes de español utilizando como base las reglas pragmáticas de uso planteadas por Lubbers Quesada y Blackwell (2009). Postulamos que la utilización de este tipo de análisis por parte del profesor de español en las producciones de sus aprendices permitirá que el docente tenga una mejor comprensión del uso que sus estudiantes realizan de este rasgo, informando y facilitando así la toma de decisiones pedagógicas.

\section{Reglas de uso del pronombre personal expreso y omitido en español (Lubbers Quesada y Blackwell, 2009)}

Lubbers Quesada y Blackwell (2009) formularon cinco reglas pragmáticas respecto del uso de pronombre personal en español por parte de hablantes nativos:

1) Con relación a la prominencia del referente: Se utiliza el pronombre omitido cuando el referente pretendido se encuentra 'en foco', es decir cuando se puede asumir que la atención de los participantes estaría puesta en el referente debido a que se le ha asignado prominencia.

(1) ...Su nombre era es Luis Enrique Trejo López y [Ø] era el más guapo de la escuela [Ø] era una escuela muy chiquita entonces y eh y este [Ø] éramos... [Ø] estuve con él cortando y volviendo [Ø] creo que en quinto semestre [Ø] fue en quinto año las chavas de sexto lo acosaban mucho porque como [Ø] era muy guapo [Ø] lo acosaban demasiado y yo soy bien celosa...1

2) Con relación al foco de la atención: Se utiliza el pronombre expreso para marcar un cambio en el foco de atención de un referente a otro, para cambiar el sujeto o tema del discurso, y para referirse a un referente diferente del que estaría 'en foco'.

1 Los ejemplos de esta sección han sido tomados de Lubbers y Blackwell (2009). 
(2) De la primera vez que [Ø] me enamoré no tiene mucho. [Ø] Fue el año pasado, de una persona que se llama Jonathan. Él, [Ø] lo conocí porque [Ø] llegó un día a saludar a uno de mis mejores amigos y mi amigo entró. Yo estaba en la Preparatoria, y mi amigo entró a una clase y él se quedó haciéndome compañia y [Ø] empezamos a platicar, y así y este.

3) Con relación a un foco de contraste: Se utiliza pronombre de sujeto expreso cada vez que se presenta información nueva o que no se presupone y cuando esta se opone a otro referente en el discurso.

(3) ...[Ø] pasamos mucho tiempo juntas además como que [Ø] es una retroalimentación, yo dependo de ella y ella de mí, [Ø] podemos hablar de de tontería y...

4) Con relación al peso pragmático: Se utiliza el pronombre de sujeto expreso "yo" con verbos correspondientes a actos de habla relativos a afirmaciones, creencias, opiniones, emociones, o conocimiento. Esto con el objeto de agregar peso pragmático a la emisión, o expresar un mayor compromiso con lo que el discurso que se está emitiendo.

(4) La primera vez que [Ø] me enamoré y yo creo que [Ø] ha sido la única,...

5) Con relación a la suspensión del juicio epistémico: No se usa sujeto expreso con verbos de suspensión de juicio epistémico (epistemic parenthetical) con el objetivo de evaluar los enunciados previos o siguientes, o para modalizar o mitigar la fuerza de un comentario evaluativo. Cabe señalar que a menudo estos verbos sí se siguen de un pronombre de sujeto expreso (en especial yo) debido a que eliminan el foco del referente, razón por la cual es necesario volver a mencionarlo.

(5) "Pues [Ø] busca, adelante". [Ø] No sé, pues [Ø] era muy cómodo de ...

\section{Metodología}

Esta investigación tiene por objeto describir el uso de los pronombres personales de primera persona singular omitidos y expresos que realizan aprendices de español de acuerdo a las reglas pragmáticas de uso postuladas por Lubbers Quesada y Blackwell (2009).

Nuestro corpus está compuesto por las transcripciones de 18 entrevistas orales semiestructuradas realizadas a aprendices de 
español. Los aprendices son estudiantes universitarios de procedencia norteamericana cuyo nivel de dominio del español se ubica en un nivel intermedio, según criterio del American Council on the Teaching of Foreign Languages (ACTFL).

El instrumento utilizado para la recolección de datos correspondió a una entrevista semiestructurada que siguió el formato de la prueba Oral Proficiency Interview (OPI) del American Council on the Teaching of Foreign Languages (ACTFL). Estas simulan conversaciones entre el aprendiz y un entrevistador entrenado para este propósito. La entrevista OPI corresponde a un procedimiento estandarizado de medición de la habilidad funcional de comunicación en el cual el entrevistador tiene como objetivo que el sujeto evaluado produzca una muestra del uso del lenguaje susceptible de evaluación.

La evaluación del uso de pronombres en este corpus se realizó por parte de la investigadora, quien en una primera etapa identificó los verbos conjugados en primera persona singular y seguidamente procedió al análisis de su correspondencia con un uso expreso u omitido del pronombre. Finalmente, cada ocurrencia se clasificó de acuerdo a las cinco reglas pragmáticas postuladas por Lubbers Quesada y Blackwell (2009).

\section{Análisis y resultados}

Para la presente investigación se identificaron en primer lugar todas las ocurrencias de verbos conjugados en primera persona singular con presencia de la forma pronominal o sin ella, es decir, las ocurrencias de pronombres de primera persona singular tanto expresos como omitidos. Concretamente, se identificó un total de 693 ocurrencias.

En una segunda instancia, contabilizamos por separado las ocurrencias de pronombres omitidos y pronombres expresos lo cual nos permitió obtener el porcentaje de ocurrencia de cada una de estas formas. Se contabilizó un total de 523 ocurrencias de pronombre omitido y 170 ocurrencias de pronombre expreso. En relación al total de ocurrencias, se observa una marcada tendencia al uso del pronombre omitido, con un $75 \%$ del total de ocurrencias, muy superior al $25 \%$ de ocurrencias de pronombre expreso (ver gráfico 1). 
Gráfico 1: Distribución general de ocurrencias de pronombres omitidos y expresos

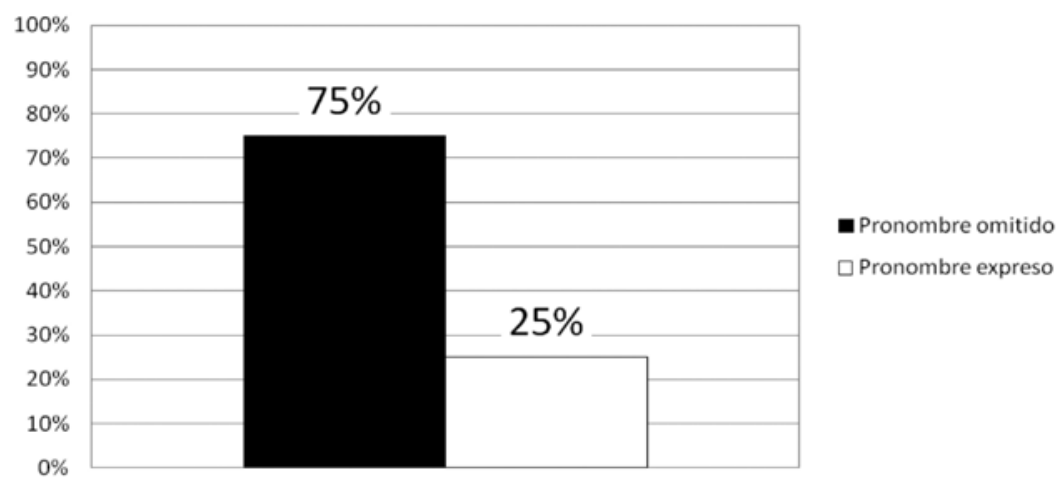

Presentamos a continuación algunos ejemplos de ocurrencias de pronombre omitido y expreso encontradas en el corpus:

(1) Pronombre omitido

(1.a) [?] He oído mucho del parque Torres del Paine. (F15)

(1.b) ...así que [?] no conozco mucha gente de mi edad... (M17)

(2) Pronombre expreso

(2.a) Yo estudio la biología amm emm, en Vermont y mmm, bueno, en XXXX College en Vermont y, sí. (F18)

(2.b) Amm, yo no he visitado una museos, pero yo he visitado una casa de Pablo Neruda que fue muy interesante, es como un barco, pero es casa. (F14)

\subsection{Análisis de uso de pronombres omitidos}

Analizamos cada ocurrencia de pronombres omitidos de acuerdo a las reglas pragmáticas 1 y 5 formuladas por Lubbers Quesada y Blackwell (2009). Identificamos como prominente (regla 1) a aquellas ocurrencias en las cuales el referente del pronombre omitido se encontraría en foco debido a su prominencia en el discurso, es decir, el referente es posible de inferir debido a que ha sido nombrado anteriormente en el discurso; debido al conocimiento mutuo de los interlocutores o; debido 
a la semántica de la oración o morfología del verbo. Se incluyeron en esta categoría todas las ocurrencias en las cuales el referente no es el mismo de la oración previa, sin embargo, la morfología del verbo no permite interpretaciones ambiguas. Nos apartamos del análisis realizado por Lubbers Quesada y Blackwell (2009), quienes consideraron estas ocurrencias como caso de cambio de foco, debido a que consideramos que la naturaleza de las entrevistas que componen nuestro corpus permite inferir que el conocimiento mutuo de los interlocutores en esta situación de comunicación específica es suficiente para que las referencias a primera persona singular obtuvieran prominencia, por lo tanto no sería necesario usar un pronombre expreso para poner en foco al referente. En esta categoría se contabilizó un total de 424 ocurrencias las cuales corresponden a un $81 \%$ del total de ocurrencias de pronombre omitido (ver gráfico 2).

Identificamos como ocurrencias de paréntesis epistémico (regla 5) a todas aquellas ocurrencias en las cuales los pronombres omitidos se combinaron con verbos de conocimiento y creencia, tales como saber, pensar, creer, etc., y que además estos funcionaban como comentarios evaluativos, modalizadores o mitigadores del contenido de las emisiones de los hablantes. En esta categoría contabilizamos 99 ocurrencias las que corresponden a un $19 \%$ del total de ocurrencias de pronombre omitido (ver gráfico 2).

Gráfico 2: Distribución de ocurrencias de pronombres omitidos con relación al uso en construcciones con referente en prominencia (regla 1) y uso en emisiones con función de paréntesis epistémico (regla 5)

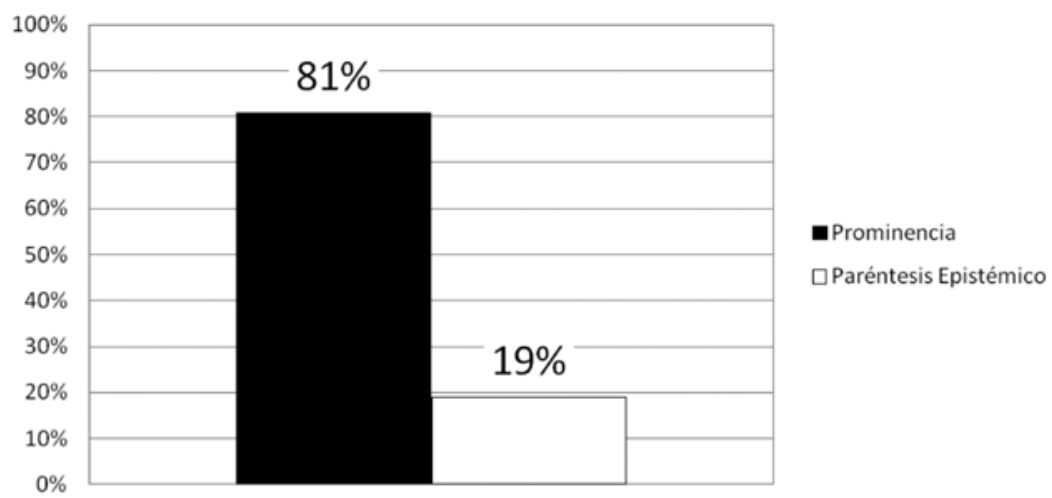


A continuación presentamos algunos ejemplos de las ocurrencias identificadas:

(3) Pronombre omitido en construcciones con referente en prominencia

(3.a) [?] Estoy bien, [?] estoy un poco enferma mmm hoy, pero [?] estoy bien y [?] estoy mejorando poco a poco. (F4)

(3.b) ...es una ciudad muy bonita, [?] no he tenido la oportunidad de visitarla mucho a la ciudad mucho, pues [?] he tenido la pierna lastimada, pero, sí, me gusta. (M17)

(4) Pronombre omitido en construcciones con función de paréntesis epistémico

(4.a) ...[?] quiero trabajar con cáncer, oncología, pero [?] no sé bien,... (M11)

(4.b) En general sí, en general [?] pienso que si, pero[?] no he visto la comida en otras partes... (F2)

\subsection{Análisis de uso de pronombres expresos}

Analizamos cada ocurrencia de pronombres expresos de acuerdo a las reglas pragmáticas 1, 2, 3, 4 y 5 formuladas por Lubbers Quesada y Blackwell (2009). En primer lugar, identificamos como prominente (regla 1) a aquellas ocurrencias en las cuales el referente del pronombre expreso se encontraría en foco debido a que el referente es posible de inferir ya que ha sido nombrado anteriormente en el discurso; debido al conocimiento mutuo de los interlocutores o; debido a la semántica de la oración o morfología del verbo. Se incluyeron en esta categoría todas las ocurrencias en las cuales el referente no es el mismo de la oración previa pero que la morfología del verbo no permite interpretaciones ambiguas. En esta categoría identificamos 131 ocurrencias, lo que corresponde al $77 \%$ del total de ocurrencias de pronombre expreso (ver gráfico 3).

En segundo lugar, identificamos como cambio de foco (regla 2) a todas aquellas ocurrencias en las cuales el pronombre expreso se utiliza para poner en foco a un referente que no es el mismo de la oración precedente. Solo identificamos en esta categoría aquellas ocurrencias en las cuales la morfología del verbo permitía una interpretación ambigua. En tanto las ocurrencias que por la morfología del verbo era posible identificar el 
referente se clasificaron como prominente (regla 1). En esta categoría no se contabilizaron ocurrencias.

Subsecuentemente, identificamos las ocurrencias en las cuales se utilizó el pronombre expreso para la oposición de referentes (regla 3). Clasificamos en esta categoría a todas aquellas ocurrencias en construcciones paralelas en las cuales aparece el uso de pronombre expreso de primera persona singular. En esta categoría, al igual que en la anterior, no se contabilizaron ocurrencias.

En cuarto lugar, se identificaron las ocurrencias en las cuales el pronombre se utilizó con función de peso pragmático, es decir, el hablante adiciona peso pragmático a su enunciado por medio de la utilización de dicho pronombre. Esto se identificó en emisiones en las cuales se utilizó el pronombre expreso en combinación con verbos de afirmación, creencia, opinión, conocimiento y emoción. En esta categoría se identificaron 34 ocurrencias, las cuales corresponden a un $20 \%$ del total de ocurrencias de pronombre expreso (ver gráfico 3).

Finalmente, identificamos como ocurrencias de paréntesis epistémico (regla 5) a todas aquellas ocurrencias en las cuales los pronombres expresos se combinaron con verbos de conocimiento y creencia, tales como saber, pensar, creer, etc., y que además estos funcionaban como comentarios evaluativos, modalizadores o mitigadores del contenido de las emisiones de los hablantes. En esta categoría identificamos 5 ocurrencias lo que corresponde a un $3 \%$ del total de ocurrencias de pronombres expresos (ver gráfico 3).

Gráfico 3: Distribución de ocurrencias de pronombres expresos en relación a uso en construcciones con referente en prominencia (regla 1), uso en construcciones con adición de peso pragmático (regla 4) y uso en emisiones con función de paréntesis epistémico (regla 5)

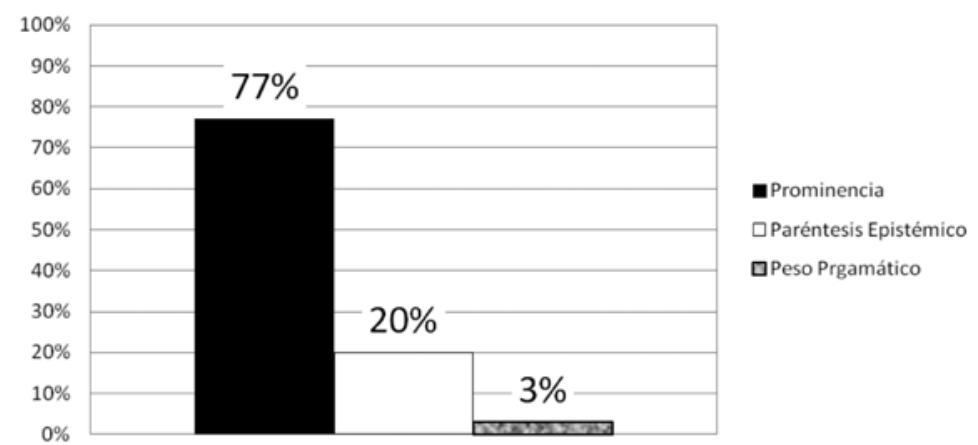


Presentamos a continuación algunos ejemplos de ocurrencias de pronombre expreso en las diferentes categorías encontradas en el corpus:

(5) Pronombre expreso en construcciones con referente en prominencia

(5.a) Yo vengo para estudiar, yo soy una estudiante extranjera.... (F4)

(5.b) Yo asisto a la Universidad de XXX² y yo estudio... (F8)

(6) Pronombre expreso en construcciones con adición de peso pragmático

(6.a) Yo conozco como dos tipos de comida mexicana, porque vivo en California, entonces tengo la comida mexicana que es más de los Estados Unidos... (F7)

(6.b) Yo quiero tomar clases de anatomía en las escuela de medicina aquí si puedo, pero si puedo, no sé si ahora. (M10)

(7) Pronombre expreso en construcciones con función de paréntesis epistémico

(7.a) ...porque yo no sé, no fue un tiempo en mi vida don... en que yo no viajo a otros lugares... (F4)

(7.b) Yo no sé, yo no sé el plan para ahora, pero quizás hay lugares que son interesantes. (F4)

\subsection{Análisis por categoría pragmática}

A continuación comparamos el número de ocurrencias de pronombres omitidos y expresos por cada categoría pragmática en la cual se contabilizaron ocurrencias en ambos usos.

\subsubsection{Uso de pronombres omitidos y expresos en construcciones con referente en prominencia}

En esta categoría se contabilizó un total de 555 ocurrencias de las cuales $424(76 \%)$ corresponden a uso combinado con pronombre omitido, y 131 (24\%) corresponden a combinación con pronombre expreso. Cabe señalar que en esta categoría se espera la utilización de pronombre omitido, en especial dada la naturaleza de las entrevistas incluidas en

2 Se suprimió la mención al nombre de la universidad en cuestión. 
el presente corpus en que no cabe ambigüedad en la interpretación del referente de los verbos en primera persona singular. El uso del pronombre expreso sería interpretado como no adecuado de acuerdo a las reglas pragmáticas señaladas en el marco referencial.

Gráfico 4: Distribución de ocurrencias en construcciones con referente en prominencia (regla 1) agrupadas por uso de pronombre omitido y expreso

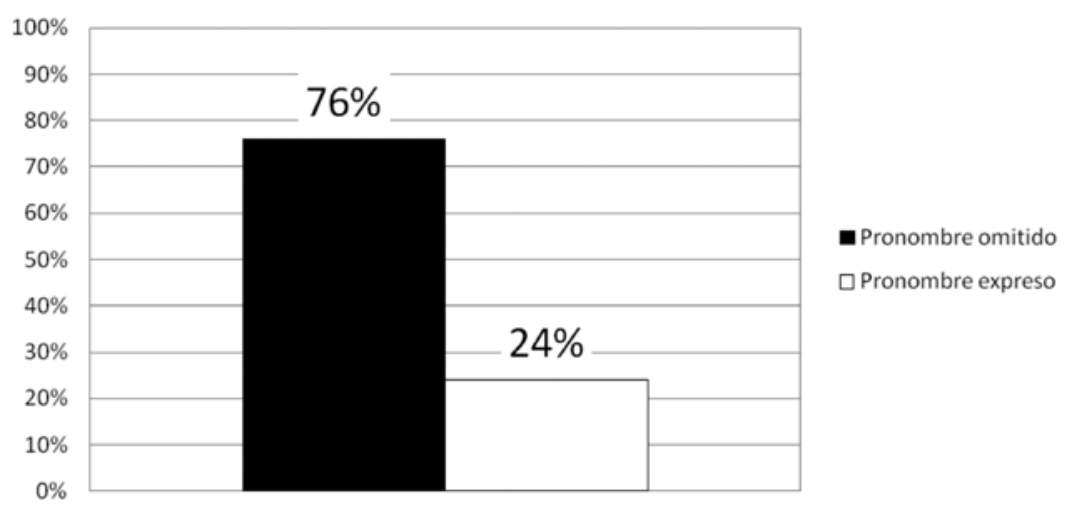

3.3.2. Uso de pronombres omitidos y expresos en construcciones en función de paréntesis epistémico

En esta categoría se contabilizó un total de 104 ocurrencias de las cuales $99(95 \%)$ corresponden a combinación con pronombres omitidos y solo $5(5 \%)$ a combinación con pronombres expresos. Cabe señalar que en esta categoría se espera la utilización de pronombre omitido, en consecuencia, el uso de pronombre expreso se interpreta como no adecuado en atención a las reglas pragmáticas señaladas en el marco referencial. 
Gráfico 5: Distribución de ocurrencias en emisiones con función de paréntesis epistémico (regla 5) agrupadas por uso de pronombre omitido (null) y expreso (overt)

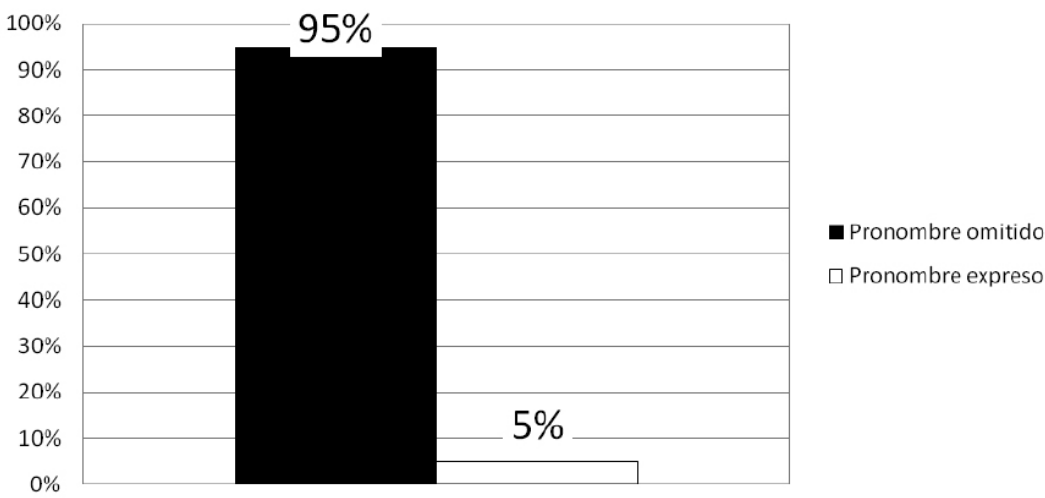

\section{Conclusiones}

Los resultados de nuestro análisis revelan un uso mayoritario de pronombres omitidos por parte de los aprendices entrevistados. Estos resultados están en consonancia con lo reportado por investigaciones anteriores en las cuales se ha observado una tendencia similar (Al-Kasey \& Pérez Leroux, 1998; Isabelli, 2004; Liceras, 1989; Lubbers Quesada \& Blackwell, 2009).

Respecto del uso de pronombres omitidos y las reglas pragmáticas estudiadas, se observa una clara tendencia a su uso en contextos de prominencia. Postulamos que este resultado puede ser consecuencia del tipo de producciones discursivas investigadas. Nuestro corpus está compuesto por respuestas a interpelaciones directas hechas por el interlocutor lo que favorece la prominencia de la persona interpelada.

En relación al uso de pronombres expresos, se observa una clara tendencia hacia la utilización de estos en contextos de prominencia del referente, sin embargo, a diferencia de lo ocurrido con el uso de pronombres omitidos, el uso en este contexto se considera como no adecuado pragmáticamente (Lubbers Quesada \& Blackwell, 2009). Esto indicaría que los hablantes representados en el corpus no habrían adquirido totalmente la competencia pragmática necesaria para el uso completamente correcto de los pronombres omitidos y expresos en este contexto. 
Respecto del uso de pronombre omitido y expreso en construcciones en función de paréntesis epistémico, se observa una clara tendencia hacia el uso de pronombre omitido. Esto se explica posiblemente debido a la internalización de frases de función epistémica las cuales no se corresponden con el uso de pronombre expreso. Nos referimos específicamente a frases como "no sé" o "pienso", la cuales otros investigadores han considerado como unanalyzed phrases (Lubbers Quesada \& Blackwell, 2009). La adquisición de estas frases se puede asimilar a la adquisición de chuncks o frases hechas las cuales se ha postulado que se internalizarían gracias a los mecanismos de aprendizaje estadístico (Saffran, Aslin \& Newport, 1996; Seidenberg \& McClelland, 1989).

Se comprueba la utilidad de la aplicación del análisis pragmático en la ocurrencia de pronombres para el área de aprendizaje de español como L2. Tal como queda de manifiesto en el desarrollo de este trabajo, el uso de este tipo de análisis en corpus de aprendices permite detectar con mayor fineza las necesidades o faltas de adecuación del uso de la lengua por parte de los aprendices, lo que redunda en datos que permiten a los docentes planificar intervenciones didácticas fundadas en las necesidades reales de los estudiantes.

Se hace necesaria una indagación más profunda respecto de los contextos lingüísticos que favorecen el uso de pronombre expreso. Como proyección de este trabajo queda indagar por ejemplo respecto de la relación del uso de pronombre expreso y oraciones en contextos adversativos. Los investigadores observaron una alta ocurrencia de este tipo de combinación, sin embargo la indagación formal y profunda de esta relación excede los límites del presente trabajo. Otra proyección del presente trabajo en este sentido, indagación de la distribución de uso de pronombre expreso y omitido en contexto de prominencia por hablante para así descartar la interferencia del uso categórico de algún hablante de uno u otro rasgo. 


\section{Referencias bibliográficas}

Aijón, M., Machado, E. \& Serrano, M. (2010). El estilo comunicativo y sus propiedades cognitivas: aplicaciones al estudio de la variación sintáctica en el español. En D. García \& M. Fumero (Eds.), Tendencias en lingüística general y aplicada (pp. 13-21). Berlín: Lang.

Aijón, M. \& Serrano, M. (2012). La posición del sujeto pronominal en cláusulas no declarativas. Onomázein, 26, 131-164.

Al-Kasey, T. \& Pérez-Leroux, A. (1998). Second language acquisition of Spanish null subjects. En S. Flynn, G. Martohardjono \& W. O'Neil (Eds.), The generative study of second language acquisition (pp. 161-183). Hillsdale: Erlbaum.

Blackwell, S. (1998). Constraints on Spanish NP anaphora: The syntactic versus the pragmatic domain. Hispania, 81, 606-618.

Blackwell, S. (2003). Implicatures on discourse: The case of Spanish NP anaphora. Amsterdam: Benjamins.

Chomsky, N. (1981). Lectures on Government and Binding: The Pisa Lectures. Holland: Foris.

Gardner, R. \& Lambert, W. (1972). Attitudes and motivation: Second language learning. Massachusetts: Newbury House.

Isabelli, C. (2004). The acquisition of null subject parameter properties in SLA: Some effects of positive evidence in a natural learning context. Hispania, 87, 150-162.

Liceras, J. (1989). On some properties of the pro-drop parameter: Looking for missing subjects in non-native Spanish. In $S$. Gass \& J. Schacter (Eds.), Linguistic perspectives on second language acquisition (pp. 109-133). Dordrecht: Foris.

Lubbers Quesada, M. \& Blackwell, S. (2009). The L2 Acquisition of Null and Overt Spanish Subject Pronouns: A Pragmatic Approach. In J. Collentine, M. García, B. Lafford \& F. Marcos (Eds.), Selected Proceedings of the 11th Hispanic Linguistic Symposium (pp. 117-130). 
Somerville: Cascadilla Proceedings Project.

Luján, M. (1999). Expresión y omisión del pronombre personal. In I. Bosque \& V. Demonte (Eds.), Gramática descriptiva de la lengua española, Vol. I (pp. 1275-1315). Madrid: Espasa Calpe.

Moreno, A. (2011). Sujetos explícitos e implícitos en la adquisición bilingüe y monolingüe del español. Lengua y Habla, 15, 73-85.

Orozco, R. \& Guy, G. (2008). El uso variable de los pronombres de sujetos: ¿qué pasa en la costa Caribe colombiana? En M. Westmoreland \& J. Thomas (Eds.), Selected Proceedings of the 4th Workshop on Spanish Sociolinguistics. Somerville, MA: Cascadilla Proceedings Project.

Ortiz, L. (2011). Spanish in contact with Haitian Creole. En M. Díaz-Campos (Ed.), The Handbook of Hispanic Sociolinguistics. Oxford: Wiley-Blackwell.

Pladevall, E. (2013). Adult L2 Spanish development of syntactic and discourse subject properties in an instructional setting. Revista Electrónica de Lingüística Aplicada, 12, 111-119.

Saffran, J., Aslin, R. \& Newport, E. (1996). Statistical learning by 8-month old infants. Science, 274, 1926-1928.

Saunders, J. (1999). Null and overt references in Spanish second language acquisition: A discourse perspective. Tesis doctoral, Universidad de Texas, Austin, Estados Unidos.

Serrano, M. \& Aijón, M. (2010a). El hablante en su discurso: Expresión y omisión del sujeto de creo. Oralia, 13, 17-38.

Serrano, M. \& Aijón, M. (2010b). La posición variable del sujeto pronominal en relación con la cortesía interactiva. Pragmalingüística, 18, 170-204.

Serrano, M. (2012). El sujeto pronominal usted/ustedes y su posición: variación y creación de estilos comunicativos. Spanish in Context, 9 (1), 109-131. 
Serrano, M. (2014). El sujeto y la subjetividad: Variación del pronombre 'yo' en géneros conversacionales y de los medios de comunicación del español de Canarias. Revista Signos, 47 (85), 321-343.

Seidenberg, M. \& McClelland, J. (1989). Visual word recognition and pronunciation: A computational model of acquisition, skilled performance, and dyslexia. In A. Galaburda (Ed.), From Neurons to Reading (pp. 255305). Cambridge: MIT Press. 